\title{
COVID-19 vaccination status within anesthesia services: navigating uncharted waters
}

\author{
Heidi V. Goldstein, MD · Joseph C. Goldstein, MD 『
}

Received: 22 January 2021/Revised: 21 February 2021 / Accepted: 22 February 2021 / Published online: 9 March 2021

(C) This is a U.S. government work and not under copyright protection in the U.S.; foreign copyright protection may apply 2021

\section{To the Editor,}

The coronavirus disease (COVID-19) pandemic continues to present major public health and economic challenges around the world, and many nations are facing yet another surge in cases with the additional concern of more communicable virus variants. ${ }^{1}$ The light at the end of the tunnel promises to come in the form of vaccines that were developed in record time. ${ }^{2}$ There is hope that swift inoculation of a significant portion of the population will lead to herd immunity and allow us to gradually return to a normal way of life. ${ }^{3}$

Since the beginning of the COVID-19 outbreak, healthcare workers have been serving diligently at the frontlines, among them anesthesiologists with their expertise in airway management. With that service comes the privilege of gaining access to the vaccines as one of the first tiers. ${ }^{4}$ This is welcome news for many in our specialty as it adds yet another layer of protection together with personal protective equipment in the fight against COVID19.

As vaccination efforts increase, many departments and essential frontline services face uncharted territory: differences in vaccination status among staff could lead

H. V. Goldstein, MD · J. C. Goldstein, MD ( $₫)$

Department of Anesthesiology, North Florida/South Georgia

Veterans Health System and the University of Florida,

Gainesville, FL, USA

e-mail: cgoldstein@anest.ufl.edu to interpersonal conflicts or ethical dilemmas. Focusing on anesthesia services in this communication, we present possible scenarios with the goal to spearhead an open dialogue leading to constructive solutions.

Consider, for example, the situation of anesthesiologists who are not able to receive a COVID-19 vaccine because of specific contraindications. Should these staff members be excluded from caring for COVID-positive patients whenever possible, as they lack the additional immunologic protection? The obvious response might be to place those that have received the full vaccination series in harm's way thus exempting the non-vaccinated, if staffing allows. This approach would be similar to how some hospital systems are practicing when excusing high risk healthcare workers from COVID-19 patient care to minimize their exposure. However, this presumes that all who are vaccinated actually develop sufficient immunity, which is not guaranteed, as immunizations can fail for a variety of reasons, ${ }^{5}$ and antibody testing for confirmation of immunity is currently not recommended by public health organizations such as the Centers for Disease Control and Prevention or the Public Health Agency of Canada. The uncertainty of adequate immunization of staff who have received the vaccine would then make an argument for not changing assignments with regards to vaccination status alone.

Let us also consider the case of anesthesiologists who have serious personal or religious objections to a COVID19 vaccine and make the decision to decline it. Can they refuse vaccination and at the same time ask to be excused from COVID-19 patient care whenever possible? And if they do not request special treatment for themselves, should leadership nevertheless exempt them from COVID19 patient care? Adding to the complexity of this topic is 
the unknown duration of protection of the various COVID19 vaccines and the related question in what time frame booster injections will have to be administered to maintain ongoing immunity. Consequently, should staff scheduling then follow the same plan that was in place before vaccination programs started irrespective of vaccination status?

As with so many issues surrounding COVID-19, this topic is still lacking guidance and will continue to evolve. There are certainly no "one-size-fits-all" answers to these scenarios. Individualized solutions will largely depend on staffing resources, the proportion of non-vaccinated to vaccinated anesthesiologists, the fraction of high-risk individuals within an anesthesia service, and the decision-making style and leadership model of the anesthesia group. Although we have discussed differences in vaccination status through the lens of anesthesia services, this topic unquestionably extends to society as a whole. We hope to raise awareness and invite open discussion as we once more collectively learn, adapt, and try to safely navigate these uncharted waters in the wake of this pandemic.

Disclosures None.

Funding statement None.
Editorial responsibility This submission was handled by Dr. Stephan K.W. Schwarz, Editor-in-Chief, Canadian Journal of Anesthesia.

\section{References}

1. Center for Infectious Disease Research and Policy. Global COVID rise continues; 50 nations report B117 variant. Available from URL: https://www.cidrap.umn.edu/news-perspective/2021/01/ global-covid-rise-continues-50-nations-report-b117-variant (accessed February 2021).

2. Kim YC, Dema B, Reyes-Sandoval A. COVID-19 vaccines: breaking record times to first-in-human trials. NPJ Vaccines 2020; DOI: https://doi.org/10.1038/s41541-020-0188-3

3. The Harvard Gazette. Fauci says herd immunity possible by fall, 'normality' by end of 2021. Available from URL: https://news. harvard.edu/gazette/story/2020/12/anthony-fauci-offers-a-timelinefor-ending-covid-19-pandemic (accessed February 2021).

4. Anesthesia Patient Safety Foundation. Protecting our Patients: APSF Recommends that Anesthesia Professionals be Included in the First Phase of Vaccinations. Available from URL: https://www. apsf.org/news-updates/protecting-our-patients-apsf-recommendsthat-anesthesia-professionals-be-included-in-the-first-phase-ofvaccinations (accessed February 2021).

5. Hinman AR, Orenstein WA, Mortimer EA Jr. When, where, and how do immunizations fail? Ann Epidemiol 1992; 2: 805-12.

Publisher's Note Springer Nature remains neutral with regard to jurisdictional claims in published maps and institutional affiliations. 